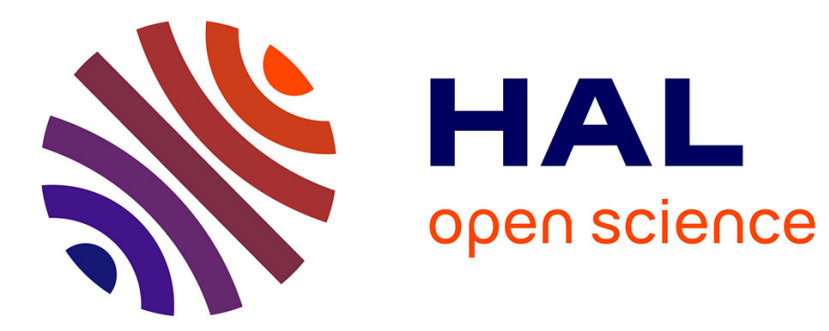

\title{
Parameter and state estimation of switched affine systems
}

Jamal Daafouz, Romain Postoyan, Pierre Riedinger

\section{To cite this version:}

Jamal Daafouz, Romain Postoyan, Pierre Riedinger. Parameter and state estimation of switched affine systems. 56th IEEE Conference on Decision and Control, CDC 2017, Dec 2017, Melbourne, Australia. hal-01652490

\section{HAL Id: hal-01652490 \\ https://hal.science/hal-01652490}

Submitted on 25 Mar 2018

HAL is a multi-disciplinary open access archive for the deposit and dissemination of scientific research documents, whether they are published or not. The documents may come from teaching and research institutions in France or abroad, or from public or private research centers.
L'archive ouverte pluridisciplinaire HAL, est destinée au dépôt et à la diffusion de documents scientifiques de niveau recherche, publiés ou non, émanant des établissements d'enseignement et de recherche français ou étrangers, des laboratoires publics ou privés. 


\title{
Parameter and state estimation of switched affine systems
}

\author{
Jamal Daafouz, Romain Postoyan, Pierre Riedinger
}

\begin{abstract}
We investigate the on-line parameter and state estimation of switched affine systems. We first adapt standard techniques available for affine (time-varying) systems to the considered class of systems, which leads to new linear matrix inequality-based conditions for the design of the estimator gains. We then explain how to derive decoupled estimators in the sense that the state and the parameter estimates are generated by cascaded switched differential equations, which is numerically more efficient and thus easier to implement in practice. A first solution is presented, which generates state estimates with jumps at the switching instants. To overcome this potential issue, two "bumpless" strategies are proposed. The first one relies on a persistency of excitation (PE) condition, while the the second one applies to a given class of switching rules and avoids the PE condition. Both estimation strategies ensure the global exponential convergence to zero of the estimation errors. The results are illustrated on a model describing the behavior of a DC-DC power converter.
\end{abstract}

\section{INTRODUCTION}

On-line parameter and state estimation is a challenging problem from a theoretical point of view and most available results with analytical convergence guarantees apply to specific classes of systems, see e.g., [12], [3], [11], [7], [9], at the exception of the recent work in [6]. The aforementioned references concentrate on smooth dynamics. In this paper, we are interested in switched affine systems, which consist of a set of affine dynamics called modes, together with a law for switching between these modes. These models are often used to describe real-world systems that are subject to known or unknown abrupt parameter changes, e.g. embedded systems in automotive industry, aerospace, and energy management. Because of the switches between the modes, results for smooth systems cannot be applied in general to these systems and adapted solutions are needed.

To the best of our knowledge, very few contributions are related to the joint parameter and state estimation of switched affine systems. In [10], the authors assume that the parameters belong to a given polytope. The problem is then formulated as a switching rule design for an auxiliary switched system whose matrices, at the equilibrium, correspond to the matrices of the switched system to be estimated. An alternative approach is simply to consider the unknown parameter as an extra state and to design a state observer for the extended system. However, the model structure is ignored

The authors are with the Université de Lorraine, CRAN, UMR 7039 and the CNRS, CRAN, UMR 7039, France firstname.name@univ-lorraine. fr. Their work was partially supported by the ANR under the grant COMPACS (ANR-13-BS03-0004$02)$. in this case and it may be difficult to design a state-observer for the obtained system.

In this study, we focus on switched affine systems for which the unknown parameters affect the affine term. We first review and adapt standard techniques, which lead to linear matrix inequality (LMI) based conditions under which a joint parameter and state estimator is synthesized. Inspired by [8], we then investigate the design of a decoupled estimator, i.e. a scheme for which the state is estimated by first generating a parameter-free state estimate, which is then corrected by a term involving the parameter estimate. Decoupled solutions are of practical interest as these ease the implementation since it leads to a cascaded estimator, see also [13] for related results on smooth affine time-varying systems. We highlight the features of the decoupled estimation problem in the context of switched systems. In particular, we show that the most simple solution leads to state estimates, with jumps at the switching instants. To avoid this phenomenon, we propose a solution using a persistency of excitation (PE) condition, which ensures the global exponential convergence of the parameter and state estimation errors to the origin. Because the PE condition may be difficult to verify, we also provide an alternative design, which does not rely on any PE property but restricts the class of switching rules. The latter scheme also ensures the global exponential convergence of the estimates. The results are applied to a DC-DC power converter, for which we aim at estimating two parameters corresponding to an unknown constant load and an unknown constant input voltage.

The paper is organized as follows. In the next section, we give the counterpart of the standard reduced-order observer and estimation theory in the case of switched affine systems. The main contribution of this paper is detailed in Section III where the specificities of the switched decoupled parameter and state estimation are highlighted. The proposed study is illustrated on a DC-DC converter example in Section IV before giving some concluding remarks in Section V.

Notations. The set composed by the $N$ first positive integers is denoted by $\mathcal{I}=\{1, \cdots, N\}$. The notation $\mathbb{I}$ stands for the identity matrix for any dimension. We use $X^{T}$ to denote the transpose of the matrix $X$, and we write $X>0(X<0)$ to denote that $X$ is positive (negative) definite. The symbol $(\bullet)$ stands for the blocks of a matrix induced by symmetry. The notation $(x, y)$ is used for $\left(x^{T}, y^{T}\right)^{T}$, for any $x \in \mathbb{R}^{n}, y \in$ $\mathbb{R}^{m}$. 


\section{JOINT STATE AND PARAMETER ESTIMATION}

We consider the switched affine system

$$
\begin{aligned}
\dot{x} & =A_{\sigma} x+B_{\sigma} u+G_{\sigma} \theta \\
y & =C_{\sigma} x
\end{aligned}
$$

where $x \in \mathbb{R}^{n}$ is the state, $u \in \mathbb{R}^{m}$ is the input, $y \in \mathbb{R}^{p}$ is the output and $\theta \in \mathbb{R}^{r}$ is an unknown constant parameter $(\dot{\theta}=0)$. The switching rule is defined by $\sigma: \mathbb{R}^{n} \rightarrow \mathcal{I}$ and is assumed to be available in real time and Lebesgue measurable.

The objective of this paper is to propose design solutions to estimate on-line $x$ and $\theta$. In this section, we concentrate on the synthesis of the so-called joint estimators. We first consider the case where $x$ is known, we then extend the results to the case where it is not.

\section{A. When the state is known}

Knowing the state, an asymptotic estimate $\hat{\theta}$ of the unknown parameter $\theta$ can be obtained using a switched full order filter given by

$$
\begin{aligned}
\dot{\hat{x}} & =A_{\sigma} x+B_{\sigma} u+G_{\sigma} \hat{\theta} \\
\hat{\theta} & =K_{\sigma}(x-\hat{x})
\end{aligned}
$$

with $\hat{x} \in \mathbb{R}^{n}$. Alternatively, a switched reduced-order filter may also be used to asymptotically estimate $\theta$ by

$$
\begin{aligned}
\dot{z} & =-K_{\sigma}\left(A_{\sigma} x+B_{\sigma} u+G_{\sigma} \hat{\theta}\right) \\
\hat{\theta} & =K_{\sigma} x+z
\end{aligned}
$$

with $z \in \mathbb{R}^{r}$. In both cases, the dynamics of the parameter estimation error $e_{\theta}:=\theta-\hat{\theta}$ is

$$
\dot{e}_{\theta}=-K_{\sigma} G_{\sigma} e_{\theta}
$$

The next proposition is dedicated to the design of $K_{\sigma}$ such that the equilibrium $e_{\theta}=0$ of system (4) is globally exponentially stable for any switching signal $\sigma$.

Theorem 1: The error dynamics (4) is globally exponentially stable for any switching signal $\sigma$ if there exist matrices $R_{i} \in \mathbb{R}^{r \times n}, i \in \mathcal{I}$, that satisfy the following LMIs

$$
G_{i}^{T} R_{i}^{T}+R_{i} G_{i}>0
$$

by taking the gain matrices $K_{i}=P^{-1} R_{i}$ where $P \in \mathbb{R}^{r \times r}$ is any positive definite matrix.

Proof: The proof is obtained using a quadratic Lyapunov function $V(e)=e^{T} P e$ with $P=P^{T}>0$ to check global exponential stability of (4) and the change of variables $K_{i}=P^{-1} R_{i}, i \in \mathcal{I}$.

The sufficient condition of Theorem 1 does not depend on the Lyapunov matrix $P$. A necessary condition for the feasibility of (5) is the observability of the pairs $\left(0, G_{i}\right)$, $i \in \mathcal{I}$, and in this case any quadratic function $V(e)=e^{T} P e$ with $P=P^{T}>0$ is a Lyapunov function that ensures global exponential stability of (4) with $K_{i}=P^{-1} R_{i}$.

\section{B. When the state is unknown}

When both the state and the parameter vectors have to be estimated, a generalization of the previous filters leads to

$$
\begin{aligned}
& \dot{\hat{x}}=A_{\sigma} \hat{x}+B_{\sigma} u+G_{\sigma} \hat{\theta}+L_{\sigma}(y-\hat{y}) \\
& \dot{\hat{\theta}}=K_{\sigma}(y-\hat{y}) \\
& \hat{y}=C_{\sigma} \hat{x},
\end{aligned}
$$

where $L_{\sigma}$ is the state estimation gain and $K_{\sigma}$ is the parameter estimation gain. Let $e_{x}:=x-\hat{x}$. We have

$$
\left[\begin{array}{c}
\dot{e}_{x} \\
\dot{e}_{\theta}
\end{array}\right]=\left[\begin{array}{cc}
A_{\sigma}-L_{\sigma} C_{\sigma} & G_{\sigma} \\
-K_{\sigma} C_{\sigma} & 0
\end{array}\right]\left[\begin{array}{c}
e_{x} \\
e_{\theta}
\end{array}\right],
$$

recall that $e_{\theta}=\theta-\hat{\theta}$. The next proposition is dedicated to the design of $L_{\sigma}$ and $K_{\sigma}$ such that the equilibrium $\left(e_{x}, e_{\theta}\right)=$ $(0,0)$ is globally exponentially stable.

Theorem 2: There exist $L_{i}$ and $K_{i}, i \in \mathcal{I}$, such that the origin of system (7) is globally exponentially stable with any arbitrary switching rule if there exist a symmetric positive definite matrix $X$, matrices $Y, M_{i}$ and $N_{i}, i \in \mathcal{I}$, of appropriate dimensions, satisfying the LMIs

$\left[\begin{array}{cc}A_{i}^{T} X+X A_{i}-M_{i} C_{i}-C_{i}^{T} M_{i}^{T} & (\bullet)^{T} \\ G_{i}^{T} X+Y A_{i}-N_{i} C_{i} & G_{i}^{T} Y^{T}+Y G_{i}\end{array}\right]<0$.

In this case, the gains $L_{i}$ and $K_{i}$ are given by

$$
\left[\begin{array}{l}
L_{i} \\
K_{i}
\end{array}\right]=\left[\begin{array}{cc}
X & Y^{T} \\
Y & Z
\end{array}\right]^{-1}\left[\begin{array}{c}
M_{i} \\
N_{i}
\end{array}\right]
$$

with $Z$ any symmetric positive definite matrix such that $Z>Y X^{-1} Y^{T}$.

Proof: The LMIs (8) are obtained using a quadratic Lyapunov function

$$
\begin{gathered}
\mathcal{V}(\zeta)=\zeta^{T} \mathcal{P} \zeta \\
\zeta=\left[\begin{array}{l}
e_{x} \\
e_{\theta}
\end{array}\right], \quad \mathcal{P}=\left[\begin{array}{cc}
X & Y^{T} \\
Y & Z
\end{array}\right]>0
\end{gathered}
$$

and the change of variables

$$
M_{i}=X L_{i}+Y^{T} K_{i}, \quad N_{i}=Z K_{i}+Y L_{i}
$$

which is equivalent to:

$$
\left[\begin{array}{c}
M_{i} \\
N_{i}
\end{array}\right]=\left[\begin{array}{cc}
X & Y^{T} \\
Y & Z
\end{array}\right]\left[\begin{array}{c}
L_{i} \\
K_{i}
\end{array}\right]
$$

The existence of the inverse in (9) is guaranteed by $Z>Y X^{-1} Y^{T}$ which is nothing than taking any $Z$ that keeps $\mathcal{P}>0$

\section{DECOUPLED STATE AND PARAMETER ESTIMATION}

\section{A. Problem statement}

The estimator obtained in Theorem 2 leads to a solution where the state estimation and the parameter estimation are coupled, in the sense that the dynamics of the state and the parameter estimates in (6) depend on each others. An alternative solution where the state estimation is decoupled from the parameter estimation is of practical interest to ease 
the implementation. In this section, we use a switched Luenberger observer for the parameter free estimation problem $\left(G_{i}=0, i \in \mathcal{I}\right)$, that is

$$
\begin{aligned}
\dot{\tilde{x}} & =A_{\sigma} \tilde{x}+B_{\sigma} u+\tilde{L}_{\sigma}(y-\tilde{y}) \\
\tilde{y} & =C_{\sigma} \tilde{x} .
\end{aligned}
$$

The variable $\tilde{x}$ is a state estimate of $x$ when $G_{i}=0$ for any $i \in \mathcal{I}$. Hence, $\tilde{x}$ is independent of the parameter estimate. Define the error $e_{\tilde{x}}:=x-\tilde{x}$. The gain $\tilde{L}_{\sigma}$ is determined such that the origin of the system

$$
\dot{e}_{\tilde{x}}=\tilde{A}_{\sigma} e_{\tilde{x}}, \quad \text { with } \quad \tilde{A}_{i}=A_{i}-\tilde{L}_{i} C_{i}, \quad i \in \mathcal{I}
$$

is globally exponentially stable. It is well known (see [5]) that if there exists a matrix $\tilde{P}=\tilde{P}^{T}>0$ and matrices $\tilde{R}_{i}$ satisfying for all $i \in \mathcal{I}$

$$
A_{i}^{T} \tilde{P}+\tilde{P} A_{i}-C_{i}^{T} \tilde{R}_{i}^{T}-\tilde{R}_{i}^{T} C_{i}<0
$$

then $\tilde{L}_{\sigma}$ with $L_{i}=\tilde{P}^{-1} \tilde{R}_{i}$ ensures that the origin of system (13) is globally exponentially stable and

$$
V_{e}\left(e_{\tilde{x}}\right)=e_{\tilde{x}}^{T} \tilde{P} e_{\tilde{x}}
$$

is a quadratic Lyapunov function. In this case, there always exists a symmetric matrix $\tilde{Q}>0$ such that, along the solutions to system (13),

$$
\dot{V}_{e}=-e_{\tilde{x}}^{T} \tilde{Q} e_{\tilde{x}} \leq 0
$$

We assume that (14) holds throughout this section and that the gains $\tilde{L}_{i}$ are given by $\tilde{P}^{-1} \tilde{R}_{i}$. Our objective is to obtain a state estimate for the switched affine system with unknown parameters using the estimate $\tilde{x}$ and a correction based on a parameter estimate $\hat{\theta}$, whose dynamics are still given by

$$
\dot{\hat{\theta}}=K_{\sigma}(y-\hat{y})
$$

and where $\hat{y}$ is expressed in terms of $\tilde{x}$ and $\hat{\theta}$.

\section{B. A first solution}

Let $T_{\sigma}$ be the set whose elements are $t_{0}=0$ and all the switching times $t_{i}$ of $\sigma$, indexed in such a way that $0=t_{0}<t_{1}<t_{2}<\ldots$. This means that between any two consecutive switching times $t_{i}$ and $t_{i+1}$, the mode $\sigma=i$ is active for all $t \in\left[t_{i}, t_{i+1}\right)$. If the set of switching times is infinite, then $\lim _{i \rightarrow+\infty} t_{i}=+\infty$.

Here, we establish LMI conditions under which a joint parameter and state estimator of the form (6) satisfies:

$$
\hat{x}=\tilde{x}+S_{\sigma} \hat{\theta}
$$

with $S_{i}, i \in \mathcal{I}$ some constant matrices to be determined. As a result, the state estimate $\hat{x}$ can be obtained from a parameterfree state estimate $\tilde{x}$ and a correcting term involving the parameter estimate $\hat{\theta}$. Indeed, one can notice that replacing $\hat{x}=\tilde{x}+S_{\sigma} \hat{\theta}$ in the estimator (6) leads to:

$$
\dot{\hat{\theta}}=K_{\sigma}(y-\hat{y})=-H_{\sigma} \hat{\theta}+K_{\sigma}\left(y-C_{\sigma} \tilde{x}\right),
$$

with

$$
H_{i}=K_{i} C_{i} S_{i}, \quad i \in \mathcal{I}
$$

This means that the decoupling is effective as it is possible to compute the state estimation $\hat{x}$ by first computing the parameter free estimate $\tilde{x}$ and then correcting this estimate by the quantity $S_{\sigma} \hat{\theta}$ with $\hat{\theta}$ obtained using (19).

Theorem 3: Suppose that there exist a symmetric matrix $X>0$, matrices $Y, M_{i}$ and $N_{i}, i \in \mathcal{I}$, of appropriate dimensions, such that, for any $i \in \mathcal{I}$,

$$
\left[\begin{array}{cc}
\tilde{A}_{i}^{T} X+X \tilde{A}_{i}-M_{i} C_{i}-C_{i}^{T} M_{i}^{T} & \bullet \\
G_{i}^{T} X+Y \tilde{A}_{i}-N_{i} C_{i} & G_{i}^{T} Y^{T}+Y G_{i}
\end{array}\right]<0 .
$$

and let $S_{i}=-\tilde{A}_{i}^{-1} G_{i}$ and $\mathcal{V}(\zeta)=\zeta^{T} \mathcal{P} \zeta$ with $\mathcal{P}=$ $\left[\begin{array}{cc}X & Y^{T} \\ Y & Z\end{array}\right]$ and $Z$ any symmetric positive definite matrix such that $Z>Y X^{-1} Y^{T}$. Then, the joint parameter and state estimator (6) with the gains

$$
\begin{aligned}
K_{i} & =\left(\mathbb{I}+S_{i}^{T} S_{i}\right)^{-1}\left[\begin{array}{ll}
S_{i}^{T} & \mathbb{I}
\end{array}\right]\left[\begin{array}{cc}
X & Y^{T} \\
Y & Z
\end{array}\right]^{-1}\left[\begin{array}{c}
M_{i} \\
N_{i}
\end{array}\right], \\
L_{i} & =\tilde{L}_{i}+S_{i} K_{i}
\end{aligned}
$$

satisfies

$$
\hat{x}=\tilde{x}+S_{\sigma} \hat{\theta}
$$

Moreover, the estimation error $\zeta$ verifies (7) for any time $t \in\left[t_{i}, t_{i+1}\right)$ and the time-derivative of $\mathcal{V}$ along the corresponding trajectories of $\zeta$, verifies for any $\left[t_{i}, t_{i+1}\right)$ such that $\zeta(t) \neq 0$

$$
\dot{\mathcal{V}}(\zeta)<0
$$

Proof: First, to have for any $t \in\left[t_{i}, t_{i+1}\right)$ the equality $\hat{x}=\tilde{x}+S_{i} \hat{\theta}, i \in \mathcal{I}$, we necessarily need to have $\dot{\hat{x}}=\dot{\tilde{x}}+S_{i} \dot{\hat{\theta}}$ for any $t \in\left[t_{i}, t_{i+1}\right)$. After replacing $\dot{\hat{x}}, \dot{\hat{\theta}}$ and $\dot{\tilde{x}}$ by their expressions (6) and (11), we derive that

$$
\left(\tilde{A}_{i} S_{i}+G_{i}\right) \hat{\theta}+\left(L_{i}-\tilde{L}_{i}-S_{i} K_{i}\right)(y-\tilde{y})=0 .
$$

The only way to guarantee that this equality is satisfied for any $i \in \mathcal{I}$ and any $t \in\left[t_{i}, t_{i+1}\right)$ is to impose $L_{i}=\tilde{L}_{i}+$ $S_{i} K_{i}$ and $S_{i}=-\tilde{A}_{i}^{-1} G_{i}, i \in \mathcal{I}$ and $t \in\left[t_{i}, t_{i+1}\right.$ ) (recall that $\tilde{A}_{i}$ is invertible as $\tilde{L}_{i}$ makes $\tilde{A}_{i}$ Hurwitz). Now one has to compute the gains $K_{i}$ and ensure that the derivative of $V(\zeta)=\zeta^{T} \mathcal{P} \zeta$ along the trajectories of (7) is strictly negative for all $t \in\left[t_{i}, t_{i+1}\right)$. Computing this derivative and using $L_{i}=\tilde{L}_{i}+S_{i} K_{i}$ and the change of variables

$$
M_{i}=\left(X S_{i}+Y^{T}\right) K_{i}, \quad \text { and } \quad N_{i}=\left(Z+Y S_{i}\right) K_{i}
$$

one obtains (20) after some algebraic manipulations. To end the proof, it remains to show that the gains $K_{i}$ are given by (21). Indeed, the previous change of variables is equivalent to

$$
\left[\begin{array}{c}
M_{i} \\
N_{i}
\end{array}\right]=\left[\begin{array}{cc}
X & Y^{T} \\
Y & Z
\end{array}\right]\left[\begin{array}{c}
S_{i} \\
\mathbb{I}
\end{array}\right] K_{i}
$$

and the existence and the unicity of the inverses in (21) is guaranteed by the fact that $\left[\begin{array}{c}S_{i} \\ \mathbb{I}\end{array}\right]$ is of full column rank and $Z>Y X^{-1} Y^{T}$ which is nothing than taking any $Z$ that keeps $\mathcal{P}>0$.

Theorem 3 provides conditions under which the joint parameter and state estimator (6) leads to an estimation 
satisfying (18). The difference with Section II-B is that the convergence of the estimation error is only ensured between two switching instants. However, at jumps, $\hat{x}$ typically jumps according to (18), which is an issue in general, and when jumps frequently occur in particular. Before explaining how to overcome this issue, we investigate the relationships between the conditions of Theorems 2 and 3. In particular, whether there are cases where the decoupled solution of Theorem 3 does not exist while the coupled solution of Theorem 2 does. In other words, are the conditions of Theorem 2 and Theorem 3 equivalent or not. The following proposition answers this important question and shows that the LMI conditions of these two theorems are equivalent; the advantage of Theorem 3 being the fact that one is given the possibility to design a decoupled state and parameter estimator.

Proposition 1: There exists a solution to the LMI conditions of Theorem 3 if and only if there exists a solution to the LMI conditions of Theorem 2.

Proof: To prove necessity, assume that the LMI conditions of Theorem 3 are feasible and denote the corresponding solution by $\tilde{X}, \tilde{Y}, \tilde{M}_{i}$ and $\tilde{N}_{i}, i \in \mathcal{I}$. These conditions read:

$$
\left[\begin{array}{cc}
\Pi_{i} & \bullet \\
G_{i}^{T} \tilde{X}+\tilde{Y} A_{i}-\left(\tilde{Y} \tilde{L}_{i}+\tilde{N}_{i}\right) C_{i} & G_{i}^{T} \tilde{Y}^{T}+\tilde{Y} G_{i}
\end{array}\right]<0
$$

with

$$
\Pi_{i}=A_{i} \tilde{X}+\tilde{X} A_{i}-\left(\tilde{X} \tilde{L}_{i}+\tilde{M}_{i}\right) C_{i}-C_{i}^{T}\left(\tilde{L}_{i}^{T} \tilde{X}+\tilde{M}_{i}\right)^{T}
$$

which means that the LMI of Theorem 2 are necessarily satisfied with

$X=\tilde{X}, \quad Y=\tilde{Y}, \quad N_{i}=\tilde{Y} \tilde{L}_{i}+\tilde{N}_{i}, \quad M_{i}=\tilde{X} \tilde{L}_{i}+\tilde{M}_{i}$

For sufficiency, assume that the LMI of Theorem 2 are feasible and denote the corresponding solution by $\bar{X}, \bar{Y}, \bar{M}_{i}$ and $\bar{N}_{i}, i \in \mathcal{I}$. This is equivalent to:

$$
\left[\begin{array}{cc}
U_{i} & \bullet \\
V_{i} & G_{i}^{T} \bar{Y}^{T}+\bar{Y} G_{i}
\end{array}\right]<0
$$

with

$$
\begin{aligned}
U_{i}= & A_{i}^{T} \bar{X}+\bar{X} A_{i}-\bar{M}_{i} C_{i}-C_{i}^{T} \bar{M}_{i}^{T}+\bar{X} \tilde{L}_{i} C_{i}- \\
& \bar{X} \tilde{L}_{i} C_{i}+C_{i}^{T} \tilde{L}_{i}^{T} \bar{X}-C_{i}^{T} \tilde{L}_{i}^{T} \bar{X}
\end{aligned}
$$

and

$$
V_{i}=G_{i}^{T} \bar{X}+\bar{Y} A_{i}-\bar{N}_{i} C_{i}+\bar{Y} \tilde{L}_{i} C_{i}-\bar{Y} \tilde{L}_{i} C_{i}
$$

which nothing than

$$
\left[\begin{array}{cc}
W_{i} & \bullet \\
G_{i}^{T} \bar{X}+\bar{Y} \tilde{A}_{i}-\left(\bar{N}_{i}-\bar{Y} \tilde{L}_{i}\right) C_{i} & G_{i}^{T} \bar{Y}^{T}+\bar{Y} G_{i}
\end{array}\right]<0
$$

where

$W_{i}=\tilde{A}_{i}^{T} \bar{X}+\bar{X} \tilde{A}_{i}-\left(\bar{M}_{i}-\bar{X} \tilde{L}_{i}\right) C_{i}-C_{i}^{T}\left(\bar{M}_{i}-\bar{X} \tilde{L}_{i}\right)^{T}$.

Hence, the LMI condition of Theorem 3 are satisfied with

$$
X=\bar{X}, \quad Y=\bar{Y}, \quad N_{i}=\bar{N}_{i}-\bar{Y} \tilde{L}_{i}, \quad M_{i}=\bar{M}_{i}-\bar{X} \tilde{L}_{i} .
$$

C. Bumpless solution under a persistency of excitation condition

The problem with the solution of Theorem 3 is that the estimation (18) may jump at the switching times as illustrated on simulations in Section IV. A way to overcome this undesirable behaviour is to consider a continuous timevarying matrix $S$, that is

$$
\hat{x}=\tilde{x}+S(t) \hat{\theta}
$$

In this case, the dynamics of the parameter estimate is still given by (19) but with

$$
H_{i}=K_{i} C_{i} S(t), \quad i \in \mathcal{I} .
$$

To impose that a joint parameter and state estimator of the form (6) satisfies (22) one has to take into account the derivative $\dot{S}(t)$ in the proof of Theorem 3 , namely

$$
\dot{\hat{x}}=\dot{\tilde{x}}+S \dot{\hat{\theta}}+\dot{S} \hat{\theta}
$$

which is equivalent to

$$
\left(\tilde{A}_{i} S_{i}+G_{i}-\dot{S}\right) \hat{\theta}+\left(L_{i}-\tilde{L}_{i}-S K_{i}\right)(y-\tilde{y})=0
$$

To guarantee that (23) holds for any $\hat{\theta}$, any $(y-\tilde{y})$ and any $i \in \mathcal{I}$ one has to impose

$$
\begin{gathered}
\dot{S}=\tilde{A}_{\sigma} S+G_{\sigma} \\
L_{\sigma}=\tilde{L}_{\sigma}+S K_{\sigma}
\end{gathered}
$$

With this choice, the conditions of Theorem 3 will become time dependent and this is not easy to handle from a practical point of view. An alternative is to use a persistency of excitation condition as stated in the next theorem.

Theorem 4: Consider the switched affine system (1) and systems (11) and (17). Let $S$ satisfying (24). There exist parameter estimation gains $K_{i}, i \in \mathcal{I}$, such that $e_{\theta}:=\theta-\hat{\theta}$ and $e_{x}:=x-\hat{x}$ (with $\hat{x}=\tilde{x}+S \hat{\theta}$ ) converge exponentially to 0 if there exist $0<\underline{\alpha} \leq \bar{\alpha}, T>0$ and symmetric positive definite matrices $\Sigma_{i}, i \in \mathcal{I}$ of appropriate dimensions such that

$$
\underline{\alpha} \mathbb{I} \leq \int_{t}^{t+T} S^{T} C_{\sigma}^{T} \Sigma_{\sigma} C_{\sigma} S d \tau \leq \bar{\alpha} \mathbb{I}
$$

In this case, the estimation gains are given by:

$$
K_{i}=\Gamma S^{T} C_{\sigma}^{T} \Sigma_{i}
$$

with $\Gamma$ any positive definite matrix.

Proof: Let

$$
e_{\tilde{x}_{s}}:=x-\tilde{x}-S \theta
$$

In view of (1), (11) and (24),

$$
\dot{e}_{\tilde{x}_{s}}=\tilde{A}_{\sigma} e_{\tilde{x}_{s}}
$$

which means that the dynamics of $e_{\tilde{x}_{s}}$ is exactly the same as the dynamics of $e_{\tilde{x}}$ given by (13) and hence $e_{\tilde{x}_{s}} \rightarrow 0$. Now, we rewrite the dynamics of the parameter estimation error $e_{\theta}=\theta-\hat{\theta}$

$$
\dot{e}_{\theta}=-\dot{\hat{\theta}}=-K_{\sigma} C_{\sigma}(x-\tilde{x}-S \hat{\theta}+S \theta-S \theta)
$$


which is nothing but

$$
\dot{e}_{\theta}=-K_{\sigma} C_{\sigma} S e_{\theta}-K_{\sigma} C_{\sigma} e_{\tilde{x}_{s}}
$$

The PE condition (26) ensures that the origin of

$$
\dot{z}=-K_{\sigma} C_{\sigma} S z
$$

is globally exponentially stable [1]. As $e_{\tilde{x}_{s}} \rightarrow 0$, we have $e_{\theta} \rightarrow 0$ that is $\hat{\theta}$ converges towards $\theta$. To end the proof, notice that

$$
x-\hat{x}=x-\tilde{x}-S \hat{\theta}+S \theta-S \theta=e_{\tilde{x}_{s}}+S e_{\theta}
$$

Finally, as $e_{\tilde{x}_{s}} \rightarrow 0, e_{\theta} \rightarrow 0$ and $S$ is bounded, we conclude that $\hat{x}$ converges toward $x$.

Compared to available results for linear time-varying systems such as those in [12], [3], here we consider switched systems and we follow the idea in [8] to impose a decoupled estimation. Theorem 4 states clearly the assumption under which such a joint decoupled estimation for switched linear systems can be obtained without bumps.

\section{Bumpless solution with no persistency of excitation con- dition}

The estimator proposed in Section III-C applies to arbitrary switching rules as long as the persistency excitation condition (26) holds. The latter may be difficult to verify. In this subsection, we propose an alternative design, which avoids checking (26) at the price of extra conditions on the switching rule. In particular, we make the next assumption.

Assumption 1: There exists a class of switching rules denoted $\mathcal{S}$ such that for any $\sigma \in \mathcal{S}$ there exist $\lambda \in \Lambda:=$ $\left\{\lambda=\left(\lambda_{1}, \ldots, \lambda_{N}\right) \in \mathbb{R}^{N} \mid \forall i \in \mathcal{I}, \lambda_{i} \geq 0, \sum_{i=1}^{N} \lambda_{i}=1\right\}$, $c_{1}, c_{2}>0$ such that any solution $S$ to (24) verifies $\left\|S_{\infty}-S(t)\right\| \leq c_{1} e^{-c_{2} t}\left\|S_{\infty}-S(0)\right\|$ for any $t \geq 0$, where $S_{\infty}:=-\tilde{A}_{\lambda}^{-1} \bar{G}_{\lambda}$ with

$$
\tilde{A}_{\lambda}:=\sum_{i=1}^{I} \lambda_{i} \tilde{A}_{i}, \quad G_{\lambda}:=\sum_{i=1}^{I} \lambda_{i} G_{i},
$$

Assumption 1 means that there exists an equilibrium matrix $S_{\infty}$ for system (1), which is globally exponentially stable. This stability property depends on the switching rule $\sigma$ and is motivated in the context of control and also by practical problems such as those related to stabilisation of power converters. The set $\mathcal{S}$ is not empty. It contains at least any stabilizing switching rule that corresponds to the sufficient conditions provided in [4] which are satisfied in our case. This can be checked by considering the convex combinations $\tilde{A}_{\lambda}, G_{\lambda}$ and defining the following polytopic affine system

$$
\dot{s}=\tilde{\mathcal{A}}_{\lambda} s+\mathcal{G}_{\lambda}
$$

with

$s=\operatorname{vec}(S), \quad g_{\lambda}:=\operatorname{vec}\left(G_{\lambda}\right), \quad \tilde{\mathcal{A}}_{\lambda}=\tilde{A}_{\lambda} \otimes \mathbb{I}, \quad \mathcal{G}_{\lambda}=g_{\lambda} \otimes \mathbb{I}$.
The fact that the matrices $\tilde{A}_{i}, i \in \mathcal{I}$, satisfy (14) means that $\forall \lambda \in \Lambda$

$$
\tilde{\mathcal{A}}(\lambda)^{T} \tilde{\mathcal{P}}+\tilde{\mathcal{P}} \tilde{\mathcal{A}}(\lambda)<0, \quad \text { with } \quad \tilde{\mathcal{P}}=P \otimes \mathbb{I} .
$$

and allows to apply Theorem 2 in [4]. As a consequence, the set $\mathcal{S}$ composed by the switching rules that stabilize the equilibrium points $s_{\infty}=-\tilde{\mathcal{A}}_{\lambda}^{-1} \mathcal{G}_{\lambda}, \lambda \in \Lambda$ is not empty and $S_{\infty}$ is the matrix counterpart of $s_{\infty}$. This steady state value is not known in general and this is why the next theorem explains how to construct the gains of the decoupled estimator without requiring the knowledge of $S_{\infty}$.

Theorem 5: Consider the switched affine system (1) and systems (11) and (17). Let $S$ satisfying (24) and suppose the following holds.

(i) Assumption 1 is satisfied.

(ii) For any $\sigma \in \mathcal{S}$, the corresponding vector $\lambda$ in Assumption 1 is such that $G_{\lambda}$ is full column rank.

There exist parameter estimation gains $K_{i}, i \in \mathcal{I}$, such that $e_{\theta}:=\theta-\hat{\theta}$ and $e_{x}:=x-\hat{x}$ (with $\hat{x}=\tilde{x}+S \hat{\theta}$ ) converge exponentially to 0 . In this case, the estimation gains are given by

$$
K_{i}=\left(\Pi+S^{T} \tilde{P} S\right)^{-1} G_{i}^{T} \tilde{P} C_{i}^{T}\left(C_{i} C_{i}^{T}\right)^{-1},
$$

with $\Pi=\Pi^{T}>0$ any symmetric positive definite matrix.

Proof: Due to space limitations, the proof can be found in the arxiv version of this paper

\section{ILLUSTRATIVE EXAMPLE}

The example represents the DC-DC power converter studied in [2]. The model is a switched affine model with a vector of unknown parameters $\theta \in \mathbb{R}^{2}$ corresponding to an unknown constant load and an unknown input voltage and it is characterized by

$$
\begin{gathered}
A_{1}=\left[\begin{array}{cc}
0 & 0 \\
0 & -0.14
\end{array}\right], \quad A_{2}=\left[\begin{array}{cc}
-3.99 & -99.97 \\
21.27 & -0.14
\end{array}\right] \\
B_{1}=\left[\begin{array}{c}
50 \\
0
\end{array}\right], \quad B_{2}=\left[\begin{array}{l}
0 \\
0
\end{array}\right], \quad C_{1}=C_{2}=\mathbb{I} \\
G_{1}=\left[\begin{array}{cc}
0 & -50 \\
-10.64 & 0
\end{array}\right], \quad G_{2}=\left[\begin{array}{cc}
1.99 & -50 \\
-10.64 & 0
\end{array}\right]
\end{gathered}
$$

The Luenberger observer (11) is designed solving LMI conditions (14) to guarantee that the error dynamics (13) is globally exponentially stable under arbitrary switching rule. The corresponding gains and Lyapunov matrix are:

$$
\begin{gathered}
\tilde{L}_{1}=\left[\begin{array}{cc}
15.5 & 0 \\
0 & 15.36
\end{array}\right], \tilde{L}_{2}=\left[\begin{array}{cc}
6 & -99.97 \\
21.27 & 10.86
\end{array}\right] \\
\tilde{P}=\left[\begin{array}{cc}
0.0406 & 0 \\
0 & 0.0397
\end{array}\right]
\end{gathered}
$$

Using Theorem 3, we obtain:

$$
S_{1}=\left[\begin{array}{cc}
0 & -3.23 \\
-0.69 & 0
\end{array}\right], \quad S_{2}=\left[\begin{array}{cc}
0.2 & -5 \\
-0.97 & 0
\end{array}\right]
$$

and:

$$
K_{1}=10^{3}\left[\begin{array}{cc}
-0.026 & -2.135 \\
-1.2914 & 0.0011
\end{array}\right]
$$




$$
K_{2}=10^{3}\left[\begin{array}{cc}
0.0412 & -1.6425 \\
-0.5747 & -0.0641
\end{array}\right]
$$

The fact that the decoupled estimate $\hat{x}(t)=\tilde{x}+S_{\sigma} \hat{\theta}$ jumps at the switching instants can be seen in Figure 1.

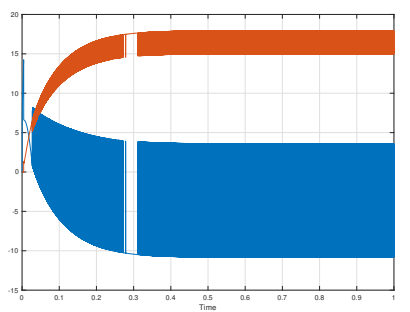

Fig. 1. $\hat{x}=\tilde{x}+S_{i} \hat{\theta}$, a decoupled estimation with bumps

Now we apply the decoupled estimation of Theorem 5. We consider $S(0)=0, \Pi=10 * \mathbb{I}$ and we use the switching rule depicted in Figure 2. This switching rule has been generated using the approach proposed in [2] and corresponds to $\lambda_{1}=$ 0.533 and $\lambda_{2}=1-\lambda_{1}$. Looking at the evolution of $S(t)$ one can see that $S(t)$ converges exactly to the equilibrium value

$$
S_{\infty}=-\tilde{A}_{\lambda}^{-1} G_{\lambda}=\left[\begin{array}{cc}
0.0850 & -3.9799 \\
-0.8121 & 0
\end{array}\right]
$$

Figure 2 shows the parameter estimation $\hat{\theta}$ for three values of the unknown parameter vector: $\theta=[3,-3]^{T}$, $\theta=[2,-3]^{T}$ and $\theta=[1,-2]^{T}$. Figure 3 shows the estimated state (solid lines), the DC-DC converter state (dotted lines), and the state of the Luenberger observer (dashed lines).
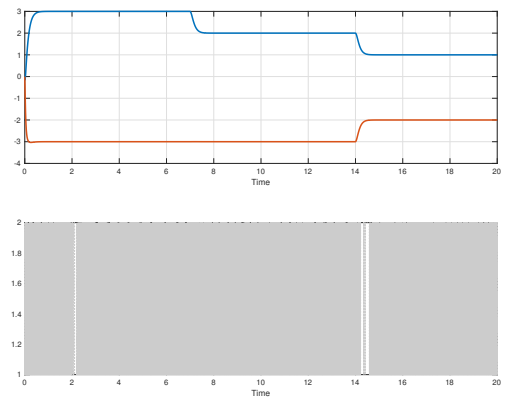

Fig. 2. $\hat{\theta}$ and the switching rule

\section{CONCLUSion}

In the general setting, the joint parameter and state estimation problem is a difficult problem. This paper shows also that the case of switched systems is challenging. Indeed, when some decoupling properties are imposed, one has to face specificities related to switching phenomena. We illustrate one of them which is the possibility of having bumps in the state estimate. The paper discusses two solutions to avoid this behavior. The first one is based on a persistency

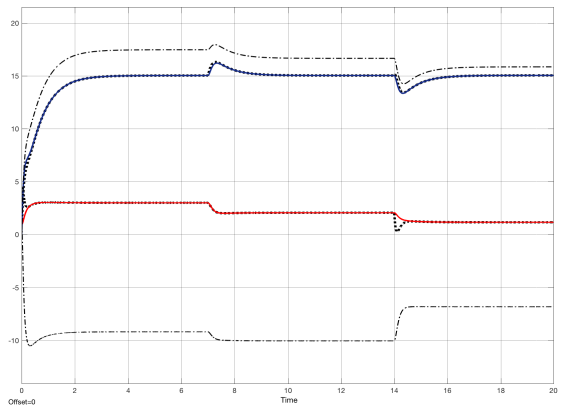

Fig. 3. DC-DC converter states $x$ in dotted lines, $\tilde{x}$ in dashed lines and $\hat{x}=\tilde{x}+S \hat{\theta}$ in solid lines

of excitation condition and the second one focuses on a particular class of switching rules and avoids this PE condition. The proposed bumpless and decoupled estimation strategies guarantee exponential convergence to zero of the estimation errors. Stability analysis of the error dynamics and on the use of the joint parameter and state estimation in the context of control of switched affine systems will be addressed in a future work.

\section{REFERENCES}

[1] B. D. O. Anderson. Exponential stability of linear equations arising in adaptive identification. IEEE Trans. on Automatic Control, 22(1):8388, 1977.

[2] G. Beneux, P. Riedinger, J. Daafouz, and L. Grimaud. Robust stabilization of switched affine systems with unknown parameters and its application to dc/dc flyback converters. In Proceedings of the IEEE American Control Conference, September 2016.

[3] G. Besancon, J. de Leon-Morales, and O. Huerta-Guevara. On adaptive observers for state affine systems and application to synchronous machines. In Decision and Control, 2003. Proceedings. 42nd IEEE Conference on, volume 3, pages 2192-2197 Vol.3, Dec 2003.

[4] P. Bolzern and W. Spinelli. Quadratic stabilization of a switched affine system about a nonequilibrium point. In American Control Conference, 2004. Proceedings of the 2004, volume 5, pages 3890-3895 vol.5, June 2004.

[5] Stephen Boyd, Laurent El Ghaoui, E. Feron, and V. Balakrishnan. Linear Matrix Inequalities in System and Control Theory. Society for Industrial and Applied Mathematics (SIAM), 1994.

[6] M.S. Chong, D. Nešić, R. Postoyan, and L. Kuhlmann. Parameter and state estimation of nonlinear systems using a multi-observer under the supervisory framework. IEEE Transactions on Automatic Control, 60(9):2336-2349, 2015.

[7] M. Farza, M. M'Saad, T. Maatoug, and M. Kamoun. Adaptive observers for nonlinearly parameterized class of nonlinear systems. Automatica, 45:2292-2299, 2009.

[8] B. Friedland. Treatment of bias in recursive filtering. IEEE Transactions on Automatic Control, 14(4):359-367, Aug 1969.

[9] David A. Haessig. On-line state and parameter estimation in nonlinear systems. In Ph.D thesis, Faculty of New Jersey Institute of Technology, 1999.

[10] L. P. Grala Pinto and A. Trofino. State and parameter estimation based on switched observers - an lmi approach. In 2014 American Control Conference, pages 3249-3254, June 2014.

[11] D. Del Vecchio and R. M. Murray. Observability and local observer construction for unknown parameters in linearly and nonlinearly parameterized systems. In American Control Conference, 2003. Proceedings of the 2003, volume 6, pages 4748-4753 vol.6, June 2003

[12] Q. Zhang. Adaptive observer for multiple-input-multiple-output (mimo) linear time-varying systems. IEEE Transactions on Automatic Control, 47(3):525-529, Mar 2002.

[13] Q. Zhang. From adaptive observers to decoupled state and parameter estimations. In Advances in Control Systems Theory and Applications, pages 242-255. USTC Press, 2009. 